\title{
TENDENCIAS ACTUALES DE LA GEOGRAFIA DEL TRANSPORTE: El el análisis de la movilidad
}

\author{
Ana Isabel Escalona Orcao \\ Universidad de Zaragoza
}

\begin{abstract}
RESUMEN: El estudio de la movilidad constituye una de las principales tendencias de la Geografía del Transporte. Tras diversas consideraciones de orden conceptual, el artículo presenta la problemática de la movilidad en los espacios urbano y regional. En ambos casos se examinan los métodos de análisis más utilizados por gébgrafos, ingenieros y planificadores del transporte.
\end{abstract}

\begin{abstract}
Study of mobility is one of the principal tendencies in Transport Geography. After some conceptual considerations this paper presents the problem of mobility in urban and regional spaces. In both cases, the methods of analysis most utilized by geographers, engineers and transport organizers are examined.
\end{abstract}

Sumario: Introducción.- Concepto de movilidad.- El Estudio de la Movilidad.Conclusión.- Bibliografía

\section{INTRODUCCION.}

Una de las secciones más interesantes de las revistas de geografía, en aquéllas que la contienen, es la que se dedica a la revisión o al comentario, por parte de algún especialista, de los avances observados en los diversos temas a partir de la literatura reciente. No es, naturalmente, el caso de este modesto artículo, que sólo pretende compartir las investigaciones de su autora en el transcurso de una estancia en el departamento de geografía de la Universidad de Sheffield 1 . Me refiero a crónicas como las últimas publicadas por Peter Rimmer sobre la evolución de la geografía del transporte en "Progress in Human Geography" (RIMMER, 1986 y 1988). Su leclura puede ser un excelente punto de partida para conocer los caminos actuales de esta rama terminal de la geografía humana y, lo que es más importante, su contribución a un mejor conocimiento del espacio a todas las escalas. En efecto, tal es la función última de los estudios sobre el movimiento de personas y mercancías por todos los modos de transporte; de los análisis de redes, de los dedicados a los movimientos de recursos e información; de los que cstablecen las relaciones entre transporte y desarrollo regional; de los que destacan las relaciones cntre el transporte y los usos de suclo, etc. Es evidentemente un campo de investigación amplísimo, lo que justifica que la presente reflexión deba de dar preferencia a algunos aspectos,

\footnotetext{
1 Dicha cstancia, que tenía como objeto estudiar los métodos y tendencias actuales de la geografia del transporte, fue posible gracias a la beca concedida por la comisión CAI/CONAI ( formada por la Caja de Ahorros de la Inmaculada y la Diputación General de Aragón). Igualmente quiero agradecer la atención que me dispensó el director del departamento de geografía. Dr. Alan Hay así como sus valiosas orientaciones durante toda la investigación. Por último vaya también mi agradecimiento al profesor Severino Escolano por sus pertinentes sugerencias tras la lectura del borrador de este artículo.
} 
como son los relacionados con el transporte de personas y, más concretamente, con el estudio de la movilidad.

Recordemos para empezar que los transportes en general, y los de personas en particular, reflejan en sus características la adaptación a un mundo en contínuo cambio (NIJKAMP y REICHMANN, 1987; POTRYKOWSKI, M. 1986) en el cual, por otra parte, cada vez son mayores los contrastes entre las sociedades y países considerados avanzados, y los países del llamado Tercer Mundo. En efecto, atrás quedó para los primeros el tiempo de la expansión de las infraestructuras de transporte, para atender una demanda creciente. Sin embargo en vastas áreas de los países menos desarrollados los medios de transporte para personas, mercancias, información, etc. son todavía escasos o inexistentes (CHATTERJEE y NIJKAMP, 1983; OWEN, 1988; HOWE y RICHARDS, 1984; BOVILL, 1978 ). Dicho de otra manera el foso entre países ricos y pobres, o por lo que se refiere al transporte, entre países "móviles" e "inmóviles" es creciente. No en vano hoy se considera que "la movilidad se ha convertido en el medio a través del cual las sociedades generan y disfrutan su propio bienestar" (NIJKAMP, 1987).

\section{EL CONCEPTO DE MOVILIDAD.}

La literatura correspondiente a la geografía del tansporte muestra que la oferta de transporte ha dejado de ser el tema central de los estudios que tienen como marco nuestro "primer" mundo. De hecho desde hace algún tiempo la preocupación de los gobernantes, e igualmente el interés de los investigadores, se encuentra en el análisis de la demanda (es decir, de las corrientes de personas y mercancías) actual y futura, a todas las escalas territoriales. En efecto, "a corto plazo no resulta ya tan neceșario como antes la creación de grandes infraestructuras "....Hoy en día el principal objetivo es el de responder lo mejor posible a una demanda más compleja en su composición y en su evolución" (MOPU, 1984). Y como decíamos, más que la demanda es la "movilidad" el objeto de tales investigaciones. Según Reichmann el concepto de movilidad supera el puramente técnico de desplazamiento, ya que incluye las causalidades y las consecuencias ligadas al propio desplazamiento. Dicho de otra manera, el acto del desplazamiento no se separa artificialmente del resto del comportamiento sino que se enlaza con las demás actividades determinadas por las motivaciones del indivíduo (REICHMANN, 1983). Se considera, por tanto, que la razón última del desplazamiento es la decisión. De esta forma la geografía del transporte entra de lleno en el campo dominado por los enfoques behavioristas.

No obstante lo anterior, de los estudios consultados se deduce la ambiguedad del concepto de movilidad, equiparado frecuentemente al de "demanda" o al de "tráfico" (BANISTER, 1985). Pero en cualquier caso creemos poder afirmar que el análisis de la demanda/movilidad es una buena aproximación al estudio de los sistemas de transporte; y que, además, confirma la calidad de la geografía del transporte para la explicación el territorio y de sus tendencias. 


\section{EL ESTUDIO DE LA MOVILIDAD.}

En un contexto en el que, como se ha dicho, la atención de los poderes publicos ha pasado de las redes a los tráficos que las soportan, el primer objetivo de los estudios de movilidad es el de explicar por qué las personas -en este caso- se desplazan, mediante la identificación de los factores que intervienen en las decisiones de desplazamiento (FISHER, 1987). En esta orientación de las investigaciones, se sigue destacando la importancia de una fuerte base teorética como fundamento del uso de modelos y métodos analíticos. Ahora bien, ¿qué métodos son los más adecuados o los que mejor se corresponden con tales fines? A este respecto la literatura existente es unánime al recomendar la sustitución de las habituales técnicas estadísticas de carácter descriptivo, por métodos basados en las ciencias sociales ${ }^{2}$, debido a su capacidad para indagar en las preferencias y motivos personales (PICKUP y TOWN, 1980; LENNTORP, 1981). Los más reputados son los modelos desagregados, que constituyen la segunda generación de modelos behavioristas (PIPKIN, 1986). No obstante su dificultad de aplicación restringe su uso habitual al medio urbano. Para el estudio de la movilidad a otras escalas, fundamentalmente a escala regional, se utilizan otros modelos derivados de la aplicación al transporte del análisis de sistemas. Un caso especial lo constituye el medio rural donde todavía la capacidad de desplazamiento puede verse limitada por la calidad de la oferta de transporte y también, dada la importancia del transporte público, por las imperfecciones del mercado. Por ello el análisis y, en su caso, la previsión de la demanda tienen como base las características, actuales y previstas, de la oferta de transporte (ANON, 1979; BANISTER, 1983 y 1985; NUTLEY, 1984).

\subsection{La movilidad en el espacio urbano.}

Uno de los elementos básicos para el funcionamiento de los espacios urbanos es, sin duda, su sistema de transporte. En efecto el crecimiento en extensión, paralelo al de la población, así como la segregación zonal justifican sin necesidad de insistir más la importancia del transporte en la "escena urbana" (TAYLOR, 1983). Es también este medio el idóneo para estudiar la movilidad de la población como factor básico de la demanda de transporte, ya que en la ciudad es donde mejor se comprende, y donde mejor se puede verificar, la influencia de hechos como la renta, el empleo, la disponibilidad de vehículo privado (sin olvidar los relacionados con el género o la edad), etc, en la capacidad de desplazamiento.

Los estudios sobre la movilidad de la población urbana, tienen ya una gran tradición en la geografía norteamericana (HANSON, 1984) pero bastante menos en Europa con la excepción de algunos países: Austria, donde destacan los trabajos del Dr. Fisher en el departamento de geografía de la universidad de Viena; Reino Unido, con las investigaciones de P. Jones en la "Transport Studies Unit" de la universidad de Oxford y las desarrolladas por H. Williams en el "Institut for Transport Studies" de la Universidad de Leeds. Pero las más conocidas serán seguramente las investigaciones dirigidas por el profesor Hägerstrand en el "Research Group for Process and Systems Analysis in Human Geography", de la universidad de Lund.

\footnotetext{
2 A pesar de que la bibliografía anglosajona emplea este término, entendemos que aquí se refiere específicamente a la sociología, psicología social, antropología y disciplinas afines.
} 
Como se sabe (POTRIKOWSKY y TAYLOR, 1984) los avances en esta materia han ido publicándose regularmente en revistas como Environment and Behaviour, Economic Geography, Environment and Planning Seric A, Geography of the Urban Environment, etc. Sin embargo una sintesis del estado actual de la cuestión apareció en "Geography of the Urban Transportation", editado por la geógrafa norteamericana Susan Hanson de la Clark University (HANSON, 1986). La tesis que subyace en la mayoría de las contribuciones es la de que el cstudio de la movilidad, o de la demanda de desplazamiento, en el espacio urbano exige la consideración de los indivíduos -y no de las zonas- como unidad de análisis. Hay por tanto un rechazo del denominado enfoque "agregado" en el que son las diferentes zonas urbanas las generadoras de desplazamientos o las destinatarias de los mismos. Dicho enfoque ha dado lugar en las pasadas décadas a la proliferación de un gran número de modelos que nutren numerosas obras de síntesis (WHITE y SENIOR, 1983; BRUTON, 1985; BLUNDEN y BLACK, 1984; etc.). Tales modelos siguen siendo muy utilizados en la práctica a pesar de que se les atribuyen numerosas insuficiencias. En efecto el enfoque agregado proporciona una imagen del funcionamiento del sistema de transporte urbano como un todo, pero "no nos ayuda a conocer algunas respuestas" relativas a las personas, a su capacidad efectiva para desplazarse y a las consecuencias de dicha capacidad (HANSON, 1986).

El enfoque desagregado, en cambio, proporciona abundante información sobre los desplazamientos de los indivíduos a lo largo del día en el espacio urbano. De sus datos deduce información detallada sobre las pautas de desplazamiento, sobre los propios indivíduos que se desplazan y sobre el contexto urbano en el que se toman las decisiones de desplazamiento. Se pueden además inferir y comprender las relaciones entre los flujos observados y los usos de suelo urbanos. No obstante tales métodos también tienen inconvenientes y el menor de ellos no es su dificultad de aplicación. La forma más frecuente (LENNTORP, 1981; ELLERGARD, 1977, etc) es la distribución entre un número variable de ciudadanos, escogidos al azar, de una especie de "diario de viaje" en cl que deben de anotar todos sus desplazamientos, así como sus características (objeto, longitud del recorrido, destino, modo de transporte utilizado, etc). Se comprende fácilmente por ello que su aplicación no haya sido muy frecuente en los circulos rclacionados con la administración pública (servicios municipales de transportes, etc); y que sus partidarios más incondicionales pertenczcan a los ámbitos académicos (HARTGEN, 1981).

\subsection{El estudio de la movilidad en el espacio regional.}

Como se acaba de señalar, la aplicación de los métodos desagregados es muy compleja, por lo que su uso se ha limitado al cspacio urbano. A escala regional los geógrafos del transporte, o quienes realizan investigaciones sobre la materia, preficren abordar el estudio de la movilidad -tanto de personas como de mercancías- en el marco ofrecido por la tcoría de sistemas. No es ésta, sin cmbargo, una novedad: cl enfoque "sistémico" aplicado al campo del transporte se desarrolla desde finales de los cincuenta, centrándose en primer lugar en los aspectos tecnológicos de los medios de transporte, y extendiéndose posteriormente a los demás elementos del sistema. Son por ello múltiples las formulaciones de "sistemas de transporte" aparecidas desde entonces. A modo de ejemplo se ha seleccionado una que puede ser representativa por haber sido ideada por el profesor israclí Shalom Reichmann, buen conocedor de la experiencia anglosajona y de la tradición francesa (Fig. 1). Scñala su autor que "como todo sistema el de transportes no funciona en el vacío; depende de un "entorno operacional" que determina tanto los resultados como las limitaciones del propio sistema" (REICHMANN, 1983). En la práctica del análisis, por tanıo, es imposible ignorar la dependencia del sistema de 
transporte respecto de dicho entomo. De la misma forma hay que asumir, tal y como se deduce del gráfico, que la movilidad dentro del sistema -expresada por el tráfico de viajeros y de mercancías- está estrechamente relacionada con los demás elementos que constituyen dicho sistema. De ahí que los estudios de movilidad intra e interregional deban de partir de su relación con la oferta de transporte, por un lado; y con el sistema socioeconómico, o sistema de actividad, por otro.

No nos referiremos a continuación a las características del estudio de la movilidad en o entre regiones (estudio que, por otra parte, recurre a las técnicas de análisis cuantitativo habituales), sino a las posibilidades que tal marco ofrece de cara a la previsión de la movilidad. Como se sabe "la claridad en la ordenación de los elementos del sistema permite descubrir relaciones entre los elementos...especificar y clarificar los procesos ... y nos proporciona un poderoso instrumento de previsión "(FRYBOURG, 1982). De tales interrelaciones se deduce que la movilidad futura es la consecuencia de los cambios que, a medio y largo plazo, ha de provocar la actual movilidad tanto en el "sistema de actividad" (distribución espacial de la población, ocupación del suelo, actividad económica, modos de vida, etc) como en la oferta de transporte. Por ello en los veinte últimos años se han ideado un gran número de modelos para la previsión de tales cambios y de sus efectos sobre la movilidad, basados todos ellos en diferentes enfoques y técnicas. La realidad muestra sin embargo que la aplicación de tales modelos no se ha extendido mucho, debido en parte a su complejidad pero también al escepticismo que se ha ido implantando entre los usuarios (tanto investigadores como planificadores) a la vista de los decepcionantes resultados obtenidos tras la aplicación de los primeros modelos, los menos elaborados. De ahí que en la práctica se siga recurriendo, para los estudios de previsión, a los convencionales modelos gravitatorios (WERNER, 1985; BRUTON, 1985; SENIOR, 1979).

No obstante existe una opinión generalizada a favor de la utilización de una segunda generación de modelos, los llamados modelos interactivos, más perfeccionados que los anteriores y a los que se les reconoce unánimemente un gran papel como ayuda a la decisión (PAULLEY, 1987).

\section{CONCLUSION.}

Tomando como hilo conductor el estudio de la movilidad, se ha presentado en las páginas anteriores una breve selección de textos representativos todos ellos de los caminos por los que actualmente discurre la geografía del transporte. Seguramente los lectores echarán en falta gran número de referencias, debido entre otras cosas a la imposibilidad de abarcar la totalidad de dichos caminos. Afortunadamente, mientras la geografía del transporte "encuentra su identidad" (GIMENEZ Y CAPDEVILA, 1986), no cesa de avanzar y de contribuir de forma valiosa a explicar el mundo en que vivimos. Hasta ahora se ha prestado mayor atención a los estudios realizados a escala nacional, regional y local, como hemos expuesto anteriormente en relación con la movilidad. No obstante de cara al futuro, los cambios tecnológicos observados en los transportes exigen la realización de estudios a escala mundial (HAY, 1985). A este respecto queremos acabar volviendo a Rimmer y a sus constataciones sobre la evolución reciente de esta rama. En efecto, nada más esperanzador (en un medio universitario como el nuestro, algo reacio a mirar fuera de su entorno) que las recientes tendencias "cosmopolitas" de la geografía del transporte. Esperamos con interés las futuras investigaciones sobre el "sistema mundial", incluyendo redes y desplazamientos intercontinentales (de personas, bienes , recursos e información); sobre las "regiones mundiales", poniendo énfasis en las relaciones 
entre transportes y desarrollo desigual a esta escala; y sobre las "ciudades mundiales", considerando las conexiones entre todas ellas mediante los transportes intercontinentales así como el impacto de tales transportes en los usos de suelo y en el medio ambiente (RIMMER, 1988). En conclusión coincidimos con Rimmer en que los geógrafos del transporte deben de desempeñar un papel fundamental en la divulgación de los cambios del espacio mundial en los próximos años.

\section{BIBLIOGRAFIA}

ANON, R. (1978) : Method for measuring rural transportation needs and demand. En Transportation Engineering $n^{2} 48$. PP. 39 a 46.

BANISTER, D. (1983) : Transport needs in rural areas. A review and proposals. En Transport Reviews, no 3, pp. 35 a 49.

BANISTER, D. (1985) : Rural transport and planning: a bibliography with abstracts. Mansell, Londres y Nueva York. 345 pp.

BANISTER, D. y HALL, P. eds. (1981) : Transport and Public Policy Planning. Mansell, 240 pp.

BEENHAKKER, H. y colbs. (1987) : Rural transport services. A guide to their planning and implementation. Intermediate Technology Publications, Londres. $379 \mathrm{pp}$.

BLUNDEN, W.R. y BLACK, J.A. (1984) : The land use/transport system. Oxford, Pergamon Press. Segunda edición. $250 \mathrm{pp}$.

BOVILL, D.N (1978) : Rural roads appraisal methods for developing countries. Transport and Road Research Laboratory Supplementary Report, $n^{9} 395.37 \mathrm{pp}$.

BRUTON, M.J. (1985) : Introduction to transportation planning Transportation. Hutchinson, Londres, tercera edición. $290 \mathrm{pp}$.

CHATTEREJEE, L. y NIJKAMP, P (1983) : Urban and regional policy analysis in developing countries. Aldershot, Gower, Londres. $258 \mathrm{pp}$.

ELLERGARD, K. y colbs. (1977) : Activity organisation and daily travels. En Economic geography, $\mathrm{n}^{2} 53$. pp. 126 a 152.

FISIIER, M. (1987) : Travel-demand modelling: A State of the Art Review. En NIJKAMP y REICHMANN ed. Transportation planning in a changing world. European Science Foundation. Gowe, pp. 171 a 186.

FRYBOURG, A. (1982) : L'analyse de systemes. En Enseignement superieur des transports, tomo uno. Conservatoire National d'Arts et Metiers/Institut de Recherche des Transports. París, pp. 45 a 73.

GIMENEZ Y CAPDEVILA, R. (1986) : La geografia de los transportes en busca de su identidad. En Geocrítica, $\mathrm{n}^{2}$ 62. Universidad de Barcelona. $63 \mathrm{pp}$.

HANSON, S. (1984) : Environmental cognition and travel behaviour. En Geography and Urban Environment, $\mathrm{n}^{2}$ 6, pp. 99 a 125.

HANSON, S. ed. (1986) : The geography of urban transportation. The Guilford Press, Nueva York y Londres. $425 \mathrm{pp}$.

HARTGEN, D. (1981) : Behavioural models in transport: perspectives, problems and prospects. En WILLIAMS, H. ed. Transport and public policy planning. Mansell, pp. 331 a 341.

HAY, A. (1973) : Transport for the Space Economy. Macmillan, 1973. $157 \mathrm{pp}$.

HAY, A. (1985) : The World as a Spatial Economic System. En Geography, pp. 97 a 105.

IIOWE, J. y RICHARDS, P. eds. (1984) : Rural roads and poverty alleviation. Intermediate Technology Publications. Londres, $191 \mathrm{pp}$.

LENNTORP, B. (1981) : A time-geographic approach to transport and public policy planning. En WILLIAMS ed.: "Transport and public policy planning". Mansell. PP. 387 a 396.

M.O.P.U. (1984) : Plan General de Carreteras 1984-1991. Memoria resumen. Secretaría General Técnica, serie documentos. Madrid, $119 \mathrm{pp}$. 
NIJKAMP, P. (1987) : " Mobility as a societal value: problems and paradoxes". En NIJKAMP y REICHMANN: Transportation planning in a changing world. European Science Foundation. Gower,pp. 73 a 92.

NIJKAMP, P. y REICHMANN, Sh. (1987) : Transportation Planning in a changing world. European Science Foundation. Gower, 292 pp.

NUTLEY, S. (1984) : Planning for rural accesibility provision: welfare, economy and equity. En Environmental and Planning, volumen $16, \mathrm{pp} .357$ a 376.

OWEN, W. (1988) : Transport and Development. Hutchinson, Londres, $168 \mathrm{pp}$.

PAULLEY, N.J. (1987) : The international study group on land-use/transport interaction: a comparison of interactive land-use/transport models. En Transportation Planning Methods. Actas del seminario C, Transport \& Planning 15th summer annual meeting. PP. 193 a 210.

PICKUP, L. (1980) : " The role of social science methodologies in transport planning". En Social aspects of transports: how to use social research in transport policy making. Transport and Road Research Laboratory supplementary report $n^{9} 698$. PP. 1 a 21.

PIPKIN, J. (1986) : Dissagregate travel models. En Geography of the urban transportation. The Guilford Press, Nueva York y Londres. PP. 179 a 206.

PLANNING AND TRANSPORT RESEARCH AND COMPUTATION - PTRC- (1987) : Transportation Planning Methods. Summer Annual Meeting September 1987, actas del seminario C. Londres, $107 \mathrm{pp}$.

POTRIKOWSKY, M. (1986) : Transport in the socialist countries. Separata de "Actas de la Reunión del grupo de trabajo Geografía del Transporte". Unión Geográfica Internacional. Conferencia Regional sobre países mediterráneos. Leon, pp. 53 a 68.

POTRIKOWSKY, M. y TAYLOR, Z. (1984) : Geografia del transporte. Ariel Geografia. Barcelona, 303 pp.

REICHMANN, Sh. (1983) : Les transports, servitude ou liberté?. Presses Universitaires de France, Paris. 197 Pp.

RIMMER, P. (1986) : Transport Geography. En Progress in Human Geography, n², pp. 396 a 405.

RIMMER, P. (1988) : Transport Geography. En Progress in Human Geography, n², pp. 270 a 279.

SENIOR, M.L. (1979) : From gravity modelling to entropy maximising. A pedagogic guide. En Progress in Human Geography, n³, pp. 175 a 210.

STOPHER, P. y MEYBOURG, A. (1976): Behavioural travel demand models. Heath. 257 pp.

WERNER, C. (1985) : Spatial Transportation modelling. Scientific Geography Series, volumen 5. Ed. Grant Ian Thrall. $90 \mathrm{pp}$.

WHITE, H. y SENIOR, M. (1983) : Transport Geography. Longman, Nueva York. 224 pp.

WILLIAMS, H. (1981) : Travel demand forecasting: and overview....En P. Hall y D. Banister (eds.) Transport and public policy planning. Mansell, $237 \mathrm{pp}$. 

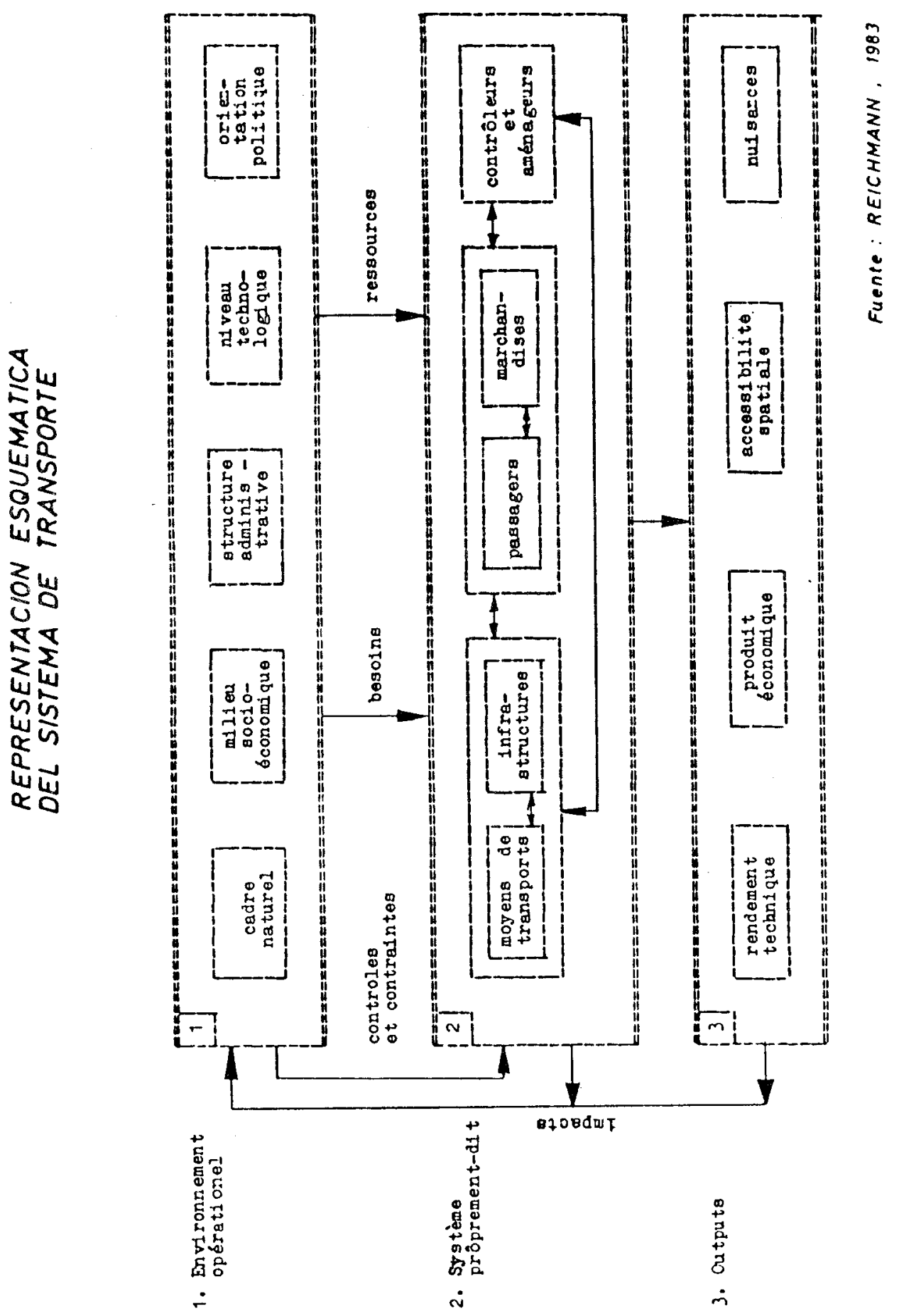